\title{
AVALIAÇÃO DO POSICIONAMENTO DA AORTA EM PACIENTES COM ESCOLIOSE IDIOPÁTICA DO ADOLESCENTE
}

\author{
EVALUATION OF THE POSITIONING OF THE AORTA IN PATIENTS WITH ADOLESCENT \\ IDIOPATHIC SCOLIOSIS
}

\section{EVALUACIÓN DE LA POSICIÓN DE LA AORTA EN PACIENTES CON ESCOLIOSIS IDIOPÁTICA DEL ADOLESCENTE}

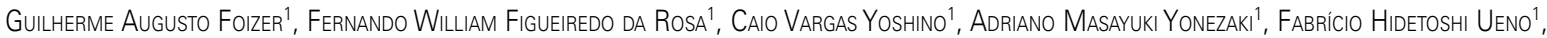
Edgar Santiago Valesin Filho', Luciano Miller Reis Rodrigues ${ }^{1}$

\begin{abstract}
RESUMO
Objetivo: Avaliar o posicionamento da aorta em pacientes com escoliose. Métodos: Foram realizados exames de imagem em pacientes ambulatoriais com escoliose idiopática do adolescente e em um grupo formado por pacientes hígidos, sendo analisados seis parâmetros: comprimento do corpo vertebral, largura, distância aorta-corpo, diâmetro da aorta, distância aorta-canal e ângulo corpo-aorta. Resultados: As curvas variaram entre T3 e L2 com ângulos de Cobb de $46^{\circ}$ e $114^{\circ}$. Pela classificação de Lenke os tipos $1 \mathrm{AN}$ e $1 \mathrm{BN}$ ocorreram em $20 \%$ dos casos, além de $1 \mathrm{CN}, 3 \mathrm{BN}, 3 \mathrm{C}+$ com 13,3 \% e 1B+, 3C- com 6,7 \%. As vértebras próximas ao ápice da curva no grupo escoliose apresentam valores superiores às do grupo controle (em T8 com média de 86,93 no grupo escoliose e média 49,07 no grupo controle $(\mathrm{p}<0,0001)$. Conclusão: Quanto mais próximo do ápice da deformidade, maior a póstero-lateralização da aorta.
\end{abstract}

Descritores: Escoliose; Aorta; Anatomia; Coluna vertebral.

\begin{abstract}
Objective: to determine the aorta positioning in patients with idiopathic scoliosis. Methods: We performed imaging studies on outpatients with adolescent idiopathic scoliosis and on a group of healthy patients, and analyzed six parameters: length of the vertebral body, width, aortic-body distance, aortic diameter, distance between spine channel and aorta and aortic-body angle. Results: Curves ranged from T3 to $\mathrm{L} 2$ with Cobb angles from $46^{\circ}$ to $114^{\circ}$. By Lenk's classification it was observed that types $1 \mathrm{AN}$ and $1 B N$ occurred in $20 \%$ of cases, 1 CN, $3 B N, 3 C+$ in $13,3 \%$ and $1 B+$, and $3 C$ - in 6,7\%. The nearest vertebrae of the apex of the curve in the scoliosis group present higher values than those of the control group (at T8 with a mean of $86.93^{\circ}$ in the scoliosis group and average of $49.07^{\circ}$ in the control group $(p<0.0001$ ). Conclusion: The nearer the apex of the deformity, the greater the lateralization and posteriorization of the aorta.
\end{abstract}

Keywords: Scoliosis; Aorta; Anatomy; Spine.

\section{RESUMEN}

Objetivo: Determinar la posición de la aorta en pacientes con escoliosis idiopática. Métodos: Se realizaron estudios de imagen en pacientes ambulatorios con escoliosis idiopática del adolescente y en un grupo de pacientes sanos, y se analizaron seis parámetros: longitud y anchura del cuerpo vertebral, distancia aorta-cuerpo, diámetro de la aorta, distancia orta-canal e ángulo cuerpo-aorta. Resultados: Las curvas van desde T3 a L2 con ángulos de Cobb de $46^{\circ}$ hasta $114^{\circ}$. En la clasificación de Lenk, los tipos 1AN 1BN ocurrieron en el 20\% de los casos, $1 C N, 3 B N, 3 C+$ en 13,3\%, 3C- y 1B+ en 6,7\%. Las vértebras cerca del vértice de la curva en el grupo escoliosis tienen valores más altos

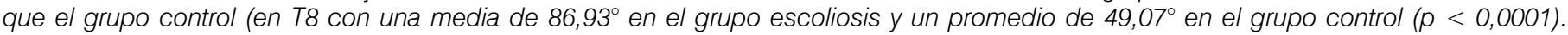
Conclusión: Cuanto más cerca del ápice de la deformidad, mayor es la lateralización y posteriorización de la aorta.

Descriptores: Escoliosis; Aorta; Anatomía; Columna vertebral.

\section{INTRODUÇÃO}

A escoliose idiopática do adolescente afeta de 1 a $3 \%$ das crianças na puberdade. É uma curvatura anormal da coluna no plano coronal associada a uma rotação no plano axial e sagital que acomete crianças principalmente próximas a puberdade. Sua etiopatogenia permanece desconhecida além de possuir uma história natural ainda não completamente bem estabelecida. ${ }^{1}$

Atualmente o uso de parafusos pediculares tem sido o tratamento de escolha para a fixação e correção das escolioses graves². O seu uso propiciou uma melhor correção devido a sua fixação em três planos permitindo reestabelecimento da biomecânica tridimensional. Os estudos realizados sobre a posição anatômica da aorta em relação ao corpo vertebral estão direcionados à técnica cirúrgica de correção através de abordagem anterior, ${ }^{3-6}$ sendo a via de acesso posterior raramente investigada nesses estudos, apesar da instrumentação posterior ser o principal método de tratamento para a maioria das curvas idiopáticas. ${ }^{1}$

Com a maior utilização dos parafusos pediculares na região torácica, ocorreu uma necessidade de maior conhecimento da relação entre a aorta torácica e a vértebra, ${ }^{6} \mathrm{com}$ o intuito de evitar lesões vasculares após instrumentação vertebral decorrente de um posicionamento inadequado do implante cirúrgico. ${ }^{7-13}$

O objetivo do presente estudo é investigar a relação entre a posição anatômica da aorta e o corpo vertebral nos diferentes padrões de magnitude de deformidade (divididos em ângulo Cobb, e classificadas de acordo com Lenke). ${ }^{14}$

1. Grupo de Coluna do Hospital Estadual Mário Covas da Faculdade de Medicina do ABC, Santo André, SP, Brasil. 


\section{MÉTODOS}

O estudo foi retrospectivo com análise de banco de imagens de pacientes com escoliose idiopática, que foram atendidos no ambulatório de patologias da coluna vertebral do Hospital Estadual Mário Covas em Santo André da Faculdade de Medicina do ABC com diagnóstico exclusivo de EIA. O critério para inclusão no estudo era apresentar alterações maiores de $20^{\circ}$ pelo método de $\mathrm{Cobb}^{15} \mathrm{em}$ radiografia realizada em posição ortostática, em incidência póstero-anterior.

No grupo controle foram incluídos pacientes que haviam sido submetidos a exames de RNM no período de janeiro de 2010 a dezembro de 2010 sem deformidade na coluna. Todos os pacientes foram submetidos a ressonância nuclear magnética (RNM) ou tomografia computadorizada (TC) e foram classificados de acordo com o método de Lenke. ${ }^{14}$

Foram avaliados posicionamento da aorta e suas relações com o corpo vertebral sendo analisadas as vértebras de T5 a L2 conforme os critérios pré estabelecidos por Sucato e Duchene ${ }^{16}$ (Figura 1 ), em imagens axiais de RNM. Somente a angulação da aorta foi modificada do método original. Sucato e Duchene estabeleceram como $0^{\circ}$ a posição em que a aorta estava estritamente lateral ao corpo vertebral e $90^{\circ}$ quando estritamente anterior. Achamos que dessa maneira poderíamos obter resultados negativos portanto definimos como $90^{\circ}$ o posicionamento anatômico estritamente lateral e $0^{\circ}$ estritamente anterior. Ângulos maiores que $90^{\circ}$ representam uma posteriorização acentuada da aorta, maior que 90 graus. (Figura 2)

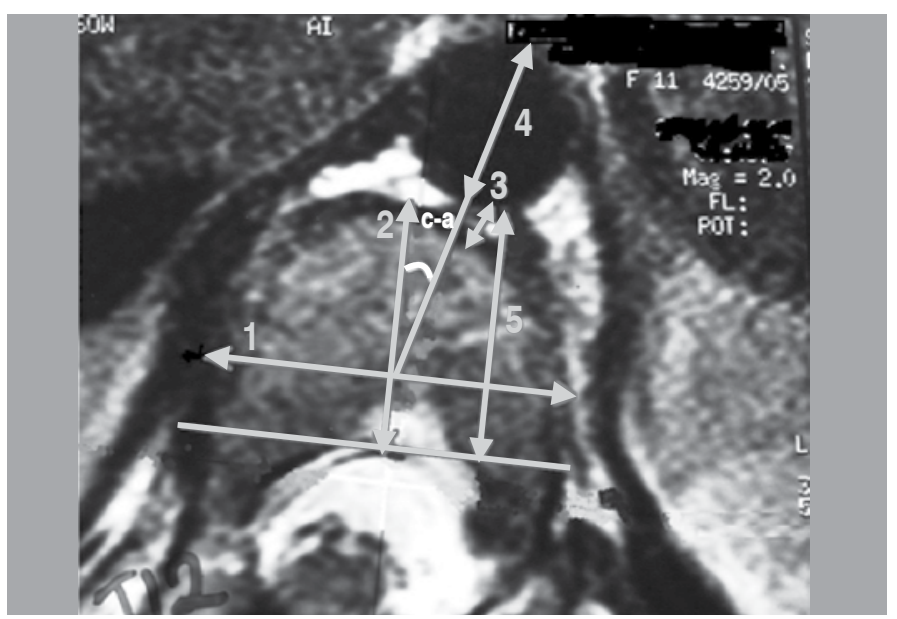

Figura 1. Parâmetros de referência.

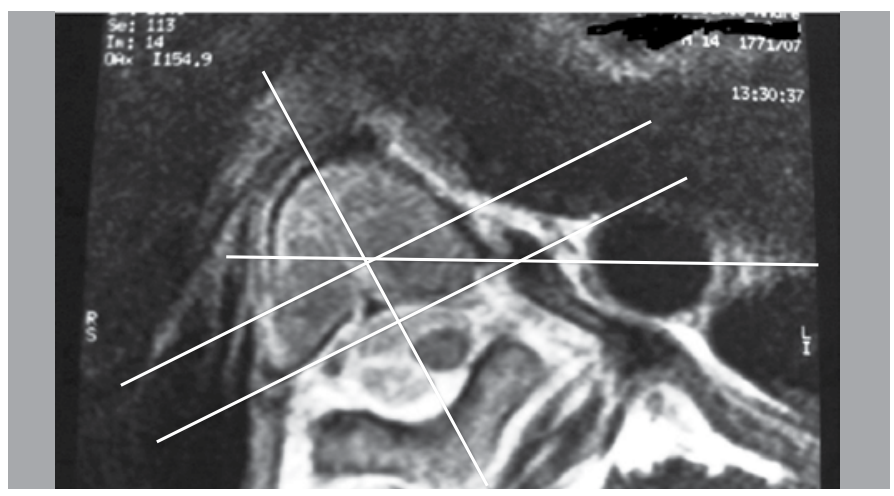

Figura 2. RNM demonstrando ângulo c-a e distância aorta-canal aumentados, com póstero-lateralização da aorta em paciente com EIA.

Os seguintes parâmetros foram medidos:

1. Largura do corpo vertebral $(\mathrm{cm})$ : distância medida entre uma córtex lateral a córtex contralateral no nível médio do corpo vertebral;

2. Profundidade anteroposterior do corpo vertebral $(\mathrm{cm})$ distância medida entre a córtex anterior e posterior no plano médio sagital;

3. Menor distância da aorta ao corpo vertebral $(\mathrm{cm})$ : menor distância medida da córtex do corpo vertebral à parede da aorta;
4. Diâmetro da aorta $(\mathrm{cm})$ : distância entre as paredes da aorta;

5. Distância entre a aorta e o canal medular (cm): distância medida do aspecto mais posterior da aorta a linha desenhada paralelamente ao aspecto anterior do canal medular;

6. Ângulo da aorta $\left(^{\circ}\right)$ : ângulo entre a linha de referência no centro do corpo vertebral e uma linha no centro da aorta, se estendendo ao centro do corpo (ângulo c-a).

Para avaliação de posicionamento de aorta em relação ao grau COBB dividimos o grupo de pacientes portadores de escoliose em: 40 a 60 graus; 61 a 80 graus e curvas maiores que 81 graus.

Os dados foram coletados por um cirurgião de coluna familiarizado com as medições e introduzidos em um sistema de dados computadorizado (Microsoft Excel 2007).

\section{ANÁLISE ESTATÍSTICA}

A escolha das medidas de tendência central e dispersão dos valores que compõem as amostras, assim como dos testes estatísticos para comparação entre as mesmas foram baseados nos tipos de distribuição. As distribuições foram definidas como paramétricas ou não paramétricas pelo teste de Kolmogorov-Smirnov segundo o programa estatístico SPSS ${ }^{\circledR}$ versão 17.0 (SPSS ${ }^{\circledR}$ Inc; llinois, USA).

Os valores obtidos pelo estudo de cada variável contínua foram organizados e descritos através da média e do desvio padrão. Para as categorizadas foram utilizadas frequências absolutas e relativas. Para a comparação entre as médias de duas populações amostrais paramétricas, foi utilizado o teste $t$ de Student. Para duas populações amostrais não paramétricas, a comparação foi feita pelo teste de Mann-Whitney e entre três ou mais populações pela aplicação do teste de Kruskall-Wallis. Adotou-se como nível de significância, a chance de ocorrência de erro tipo I menor que $5 \%(p<0,05)$.

\section{RESULTADOS}

Foram avaliados 15 pacientes no grupo escoliose com idades variando entre 13 a 20 anos (média 15,27 anos DP 2.187); o grupo controle foi caracterizado por 15 pacientes com idades entre 17 a 57 anos (média 38.80 anos DP 13.219). (Tabela 1)

Os pacientes do grupo escoliose foram classificados de acordo com Lenke ${ }^{14}$. Foi observada uma maior prevalência do tipo $1 \mathrm{AN}$ e $1 \mathrm{BN}$ com $20 \%$ dos casos além de $1 \mathrm{CN}, 3 \mathrm{BN}, 3 \mathrm{C}+\operatorname{com} 13,3 \%$ e 1B+, 3C- COM 6,7\%. (Tabelas 2 e 3)

$\mathrm{O}$ tamanho da curva variou entre T3 a L2 com ângulos Cobb de $46^{\circ}$ e $114^{\circ}$ e a vértebra apical com maior frequência no nível T8 (10 pacientes) e T9 (5 pacientes). (Tabela 3 )

Foi avaliado ainda o ângulo formado entre o maior diâmetro da aorta e o corpo vertebral (ângulo c-a). A divisão dos pacientes pelo ângulo da curva na escoliose apresentou cinco pacientes para cada grupo, com média de 85,80 graus do ângulo c-a no grupo com curvas entre 40 a $60^{\circ}, 88,8$ graus de c-a nos pacientes com curvas entre 61 a 80 graus e $112^{\circ}$ nos pacientes com escolioses maiores de $81^{\circ}$. Nesta analise não encontramos variações estatisticamente significativas, com valor $p$ encontrado foi 0,127 . (Tabela 4)

Tabela 1. Idade média grupo escoliose e grupo controle

\begin{tabular}{c|c|c|c|c|c}
\hline & $\begin{array}{c}\text { Grupo } \\
\text { (1=caso 2 =controle) }\end{array}$ & N & Média & $\begin{array}{c}\text { Desvio } \\
\text { padrão }\end{array}$ & $\begin{array}{c}\text { Erro padrão } \\
\text { da média }\end{array}$ \\
\hline Idade & 1 & 15 & 15.27 & 2.187 & .565 \\
\cline { 2 - 6 } (anos) & 2 & 15 & 38.80 & 13.219 & 3.413 \\
\hline
\end{tabular}

Tabela 2. Prevalência dos casos de acordo com a classificação de Lenke ${ }^{14}$.

\begin{tabular}{c|c|c|c|c}
\hline & Frequência & Porcentagem & $\begin{array}{c}\text { Porcentagem } \\
\text { válida }\end{array}$ & $\begin{array}{c}\text { Porcentagem } \\
\text { acumulativa }\end{array}$ \\
\hline $1 \mathrm{AN}$ & 3 & 20.0 & 20.0 & 20.0 \\
\hline $1 \mathrm{~B}+$ & 1 & 6.7 & 6.7 & 26.7 \\
\hline $1 \mathrm{BN}$ & 3 & 20.0 & 20.0 & 46.7 \\
\hline $1 \mathrm{CN}$ & 2 & 13.3 & 13.3 & 60.0 \\
\hline $2 \mathrm{C}+$ & 1 & 6.7 & 6.7 & 66.7 \\
\hline $3 \mathrm{BN}$ & 2 & 13.3 & 13.3 & 80.0 \\
\hline $3 \mathrm{C}-$ & 1 & 6.7 & 6.7 & 86.7 \\
\hline $3 \mathrm{C}+$ & 2 & 13.3 & 13.3 & 100.0 \\
\hline Total & 15 & 100.0 & 100.0 & \\
\hline
\end{tabular}


Tabela 3. Descritivo dos pacientes incluídos no estudo.

\begin{tabular}{|c|c|c|c|c|}
\hline Paciente & Idade (anos) & Cobb & $\begin{array}{l}\text { Ângulo aorta no ápice } \\
\text { e vértebra apical }\end{array}$ & Lenke \\
\hline 1 & 13 & T6-L1 90 & $80^{\circ}-\mathrm{T} 9$ & $2 \mathrm{C}+$ \\
\hline 2 & 17 & T5-L2 114 & $125^{\circ}$-T9 & $1 \mathrm{CN}$ \\
\hline 3 & 13 & T6-T12 52 & $58^{\circ}-78$ & $1 \mathrm{AN}$ \\
\hline 4 & 16 & T5-T11 53० & $107^{\circ}-\mathrm{T} 8$ & $3 \mathrm{C}+$ \\
\hline 5 & 14 & T6-L3 95 & $116^{\circ}$-T9 & $3 \mathrm{BN}$ \\
\hline 6 & 13 & T4-L1 46 & $82^{\circ}-\mathrm{T9}$ & $1 \mathrm{BN}$ \\
\hline 7 & 16 & T3-L2 64 & $82^{\circ}$-T9 & $1 \mathrm{~B}+$ \\
\hline 8 & 14 & T5-T12 95० & $130^{\circ}-\mathrm{T} 8$ & $3 \mathrm{C}-$ \\
\hline 9 & 20 & T5-T12 50 & $94^{\circ}-18$ & $1 \mathrm{AN}$ \\
\hline 10 & 15 & T6-T12 50 & $88^{\circ}-18$ & $3 \mathrm{BN}$ \\
\hline 11 & 18 & T5-T11 62 & $100^{\circ}-T 8$ & $1 \mathrm{AN}$ \\
\hline 12 & 15 & T5-T12 69० & $82^{\circ}-18$ & $1 \mathrm{BN}$ \\
\hline 13 & 18 & T5-T11 65० & $100^{\circ}-T 8$ & $3 \mathrm{C}+$ \\
\hline 14 & 14 & T5-L1 102 & $112^{\circ}-18$ & $1 \mathrm{CN}$ \\
\hline 15 & 13 & T5-T12 78 & $80^{\circ}-\mathrm{T} 8$ & $1 \mathrm{BN}$ \\
\hline
\end{tabular}

Tabela 4. Comparação entre Cobb e ângulo da aorta no ápice.

\begin{tabular}{c|c|c|c|c}
\hline \multirow{2}{*}{ Coob } & \multirow{2}{*}{$\mathbf{n}$} & \multicolumn{2}{|c|}{ Ângulo aorta } & \multirow{2}{*}{ (Kruskal-Wallis) } \\
\cline { 3 - 4 } & & Média & Desvio-Padrão & \\
\hline $40-60$ graus & 5 & 85,80 & 18,089 & \multirow{2}{*}{0,127} \\
\hline $61-80$ graus & 5 & 88,80 & 10,257 & \\
\hline$>81$ graus & 5 & 112,60 & 19,565 & \\
\hline
\end{tabular}

Verificou-se que o ângulo nas vértebras próximas ao ápice da curva no grupo com escoliose apresentam valores superiores aos do grupo controle ( em T8 com média de 86,93 DP 12,76 no grupo com escoliose e média 49,07 no grupo controle com um DP 18,71 $\mathrm{p}<0,0001$, além de T9 com média de $91,40^{\circ}$ no grupo escoliose DP 17,39 e grupo controle com ângulo médio de 39,87 DP 20,16 $p<0,0001$ ). (Tabela 5)

$\mathrm{Na}$ análise comparativa entre os valores médios obtidos no estudo do ângulo c-a em grupos de pacientes portadores de EIA e não portadores, observamos uma diminuição dos valores no grupo controle das vértebras proximais para as distais (de T5 a L1) enquanto no grupo com escoliose ocorreu um aumento até o ápice com diminuição para as vértebras distais. (Tabela 5)

$\mathrm{Na}$ análise da distância aorta-canal observamos entre o grupo de pacientes portadores de EIA os menores valores médios compreendidos nos níveis mais apicais, principalmente em T8, média de 0,07 (os ápices das curvas ocorreram em 66,6\% dos pacientes em T8 e 33,3\% dos pacientes em T9), sugerindo que na EIA, até ao ápice da curva ocorre a posteriorização da aorta. Observamos nos valores diferenças estatisticamente significativas $(p=0,006)$ na análise comparativa com pacientes do grupo controle, neste nível. Assim, em T8 observamos a menor distância entre canal aorta e neste nível houve a maior variação em relação aos valores obtidos em pacientes normais. (Tabela 6)

A aproximação da aorta ao corpo vertebral foi avaliada pela distância entre essas estruturas. Os valores mostram que no ápice das curvas, ocorre uma aproximação da aorta em relação ao corpo vertebral, inversamente ao que se observa na análise dos dados do grupo controle, que apresentam valores crescentes (T8 com média de 0,49 cm de distância do corpo e DP 0,41 no grupo escoliose e média de 1,78 cm e DP 0,90 no grupo controle com $\mathrm{p}<0,0001$ ). (Tabela 7)

Não observamos variações estatisticamente significativas na análise comparativa entre o valor do diâmetro do corpo vertebral em pacientes do grupo de escoliose e controle, exceto em T5 e L1. (Tabela 8)

$\mathrm{Na}$ análise do comprimento do corpo vertebral, observamos valores estatisticamente diferentes na analise comparativa de valores de pacientes com escoliose e controle nos níveis exceto no nível T5, com valores médios inferiores no grupo escoliose em todos os demais níveis.

O diâmetro da aorta foi avaliado comparativamente sendo encontrado valores relativos divergentes principalmente em níveis mais proximais o que pode ser observado pelo $p$ em T5,T6,T7. (Tabela 9)
Tabela 5. Comparações entre grupo escoliose e controle quanto ao ângulo corpo-aorta.

\begin{tabular}{c|c|c|c|c|c|c|c|c}
\hline \multicolumn{10}{c}{ Ângulo c-a em graus } \\
\hline \multirow{2}{*}{ Vértebra } & \multicolumn{1}{|c}{ Grupo Caso } & \multicolumn{2}{c}{ Grupo Controle } & \multicolumn{2}{c}{ Estatística } \\
\cline { 2 - 9 } & N & Média & DP & n & Média & DP & P & Teste \\
\hline T5 & 10 & 59,70 & 28,43 & 11 & 61,36 & 12,51 & 0,672 & Mann-Whitney \\
\hline T6 & 15 & 72,40 & 21,32 & 15 & 60,67 & 14,32 & 0,088 & T-Student \\
\hline$T 7$ & 15 & 80,13 & 14,65 & 15 & 53,93 & 18,30 & $<0,0001$ & T-Student \\
\hline T8 & 15 & 86,93 & 12,76 & 15 & 49,07 & 18,71 & $<0,0001$ & T-Student \\
\hline T9 & 15 & 91,40 & 17,39 & 15 & 39,87 & 20,16 & $<0,0001$ & T-Student \\
\hline$T 10$ & 15 & 74,40 & 24,90 & 15 & 29,67 & 19,81 & $<0,0001$ & T-Student \\
\hline T11 & 15 & 60,93 & 31,91 & 15 & 21,93 & 12,61 & $<0,0001$ & Mann-Whitney \\
\hline T12 & 12 & 53,58 & 37,70 & 2 & 5,00 & 7,07 & 0,104 & T-Student \\
\hline L1 & 4 & 81,25 & 40,90 & 1 & 8,00 & - & 0,208 & T-Student \\
\hline L2 & 2 & 79,00 & 0,00 & 0 & - & - & - & - \\
\hline
\end{tabular}

Tabela 6. Comparações entre grupo caso e controle quanto à distância do canal.

\begin{tabular}{|c|c|c|c|c|c|c|c|c|}
\hline \multirow{2}{*}{ Vértebra } & \multicolumn{3}{|c|}{ Grupo Caso } & \multicolumn{3}{|c|}{ Grupo Controle } & \multicolumn{2}{|c|}{ Estatística } \\
\hline & $\mathbf{n}$ & Média & DP & $\mathbf{n}$ & Média & DP & $\mathbf{P}$ & Teste \\
\hline T5 & 10 & 0,46 & 0,45 & 11 & 0,47 & 0,14 & 0,777 & Mann-Whitney \\
\hline T6 & 15 & 32 & 0,42 & 15 & 0,32 & 0,20 & 0,991 & T-Student \\
\hline $\mathrm{T7}$ & 15 & 0,18 & 0,30 & 15 & 0,39 & 0,31 & 0,082 & T-Student \\
\hline T8 & 15 & 0,07 & 0,19 & 15 & 0,34 & 0,29 & 0,006 & T-Student \\
\hline T9 & 15 & 0,33 & 0,43 & 15 & 0,30 & 0,30 & 0,808 & T-Student \\
\hline T10 & 15 & 67 & 0,88 & 15 & 0,21 & 0,20 & 0,797 & Mann-Whitney \\
\hline & 15 & 1,03 & 1,37 & 15 & 0,18 & 0,14 & 0,042 & Mann-Whitney \\
\hline T12 & 12 & 1,01 & 1,31 & 2 & 0,31 & 0,06 & 0,477 & T-Student \\
\hline $\mathrm{L} 1$ & 4 & 0,22 & 1,65 & 1 & 0,26 & - & 0,984 & T-Student \\
\hline L2 & 2 & 0,00 & 0,00 & 0 & & & & \\
\hline
\end{tabular}

Tabela 7. Comparações entre grupo caso e controle quanto à distância aorta-corpo.

\begin{tabular}{c|c|c|c|c|c|c|c|c}
\hline \multirow{2}{*}{ Vértebra } & \multicolumn{3}{|c|}{ Grupo Caso } & \multicolumn{2}{c|}{ Grupo Controle } & \multicolumn{2}{c}{ Estatística } \\
\cline { 2 - 9 } & n & Média & DP & n & Média & DP & P & Teste \\
\hline $\mathrm{T} 5$ & 10 & 0,79 & 0,46 & 11 & 0,86 & 0,42 & 0,721 & T-Student \\
\hline $\mathrm{T} 6$ & 15 & 0,76 & 0,54 & 15 & 1,07 & 0,78 & 0,219 & T-Student \\
\hline $\mathrm{T} 7$ & 15 & 0,59 & 0,63 & 15 & 1,54 & 0,90 & 0,002 & T-Student \\
\hline $\mathrm{T} 8$ & 15 & 0,49 & 0,41 & 15 & 1,78 & 0,90 & $<0,0001$ & Mann-Whitney \\
\hline $\mathrm{T} 9$ & 15 & 0,61 & 0,34 & 15 & 2,45 & 1,17 & $<0,0001$ & Mann-Whitney \\
\hline $\mathrm{T} 10$ & 15 & 0,55 & 0,40 & 15 & 2,48 & 0,85 & $<0,0001$ & T-Student \\
\hline $\mathrm{T} 11$ & 15 & 0,55 & 0,37 & 15 & 3,22 & 0,88 & $<0,0001$ & Mann-Whitney \\
\hline $\mathrm{T} 12$ & 12 & 0,63 & 0,60 & 2 & 3,51 & 0,47 & $<0,0001$ & T-Student \\
\hline $\mathrm{L} 1$ & 4 & 1,11 & 0,52 & 1 & 3,68 & - & 0,022 & T-Student \\
\hline $\mathrm{L} 2$ & 2 & 1,62 & 0,00 & 0 & - & - & - & - \\
\hline $\mathrm{L} 2$ & 2 & 3,75 & 0,00 & 0 & - & - & - & - \\
\hline
\end{tabular}

Tabela 8. Comparações entre grupo caso e controle quanto ao comprimento.

\begin{tabular}{|c|c|c|c|c|c|c|c|c|}
\hline \multirow{2}{*}{ Vértebra } & \multicolumn{3}{|c|}{ Grupo Caso } & \multicolumn{3}{|c|}{ Grupo Controle } & \multicolumn{2}{|c|}{ Estatística } \\
\hline & $\mathbf{n}$ & Média & DP & $\mathbf{n}$ & Média & DP & $\mathbf{P}$ & Teste \\
\hline T5 & 10 & 1,95 & 0,55 & 11 & 2,40 & 0,31 & 0,129 & Mann-Whitney \\
\hline T6 & 15 & 2,06 & 0,50 & 15 & 2,83 & 0,70 & 0,002 & T-Student \\
\hline $\mathrm{T7}$ & 15 & 2,20 & 0,52 & 15 & 2,70 & 0,31 & 0,003 & T-Student \\
\hline T8 & 15 & 2,40 & 0,35 & 15 & 2,81 & 0,31 & 0,003 & T-Student \\
\hline T9 & 15 & 2,49 & 0,41 & 15 & 3,01 & 0,30 & $<0,0001$ & T-Student \\
\hline T10 & 15 & & 0,38 & 15 & 3,00 & 0,45 & 0,002 & T-Student \\
\hline & 15 & & 0,37 & 15 & 3,02 & 0,41 & 0,013 & T-Student \\
\hline T12 & 12 & 2,48 & 0,27 & 2 & 2,97 & 0,37 & 0,040 & T-Student \\
\hline $\mathrm{L} 1$ & 4 & 2,37 & 0,18 & 1 & 3,28 & - & 0,020 & T-Student \\
\hline & 2 & 2,31 & 0,00 & 0 & & & & \\
\hline
\end{tabular}

\section{DISCUSSÃO}

Ao compararmos a posição da aorta em indivíduos portadores de escoliose com curva torácica principal direita com indivíduos normais, observamos que a aorta encontra-se posicionada mais posteriormente e mais próxima da vértebra devido a rotação vertebral e anomalia morfológica no plano vertical, o que segue os dados observados na literatura. ${ }^{6,17,18}$ A análise do ângulo c-a visa demonstrar se ocorreria uma alteração morfológica que pudesse colocar em risco as estruturas adjacentes durante a via de acesso ou durante a colocação 
Tabela 9. Comparações entre grupo caso e controle quanto ao diâmetro da aorta.

\begin{tabular}{c|c|c|c|c|c|c|c|c}
\hline \multirow{2}{*}{ Vértebra } & \multicolumn{3}{|c|}{ Grupo Caso } & \multicolumn{3}{c|}{ Grupo Controle } & \multicolumn{2}{c}{ Estatística } \\
\cline { 2 - 9 } & n & Média & DP & n & Média & DP & P & Teste \\
\hline $\mathrm{T} 5$ & 10 & 1,67 & 0,27 & 11 & 2,56 & 0,47 & $<0,0001$ & T-Student \\
\hline $\mathrm{T} 6$ & 15 & 1,48 & 0,33 & 15 & 2,28 & 0,40 & $<0,0001$ & T-Student \\
\hline $\mathrm{T} 7$ & 15 & 1,56 & 0,32 & 15 & 2,17 & 0,36 & $<0,0001$ & T-Student \\
\hline $\mathrm{T} 8$ & 15 & 1,58 & 0,39 & 15 & 2,16 & 0,46 & 0,001 & T-Student \\
\hline $\mathrm{T} 9$ & 15 & 1,77 & 0,25 & 15 & 2,08 & 0,41 & 0,024 & Mann-Whitney \\
\hline $\mathrm{T} 10$ & 15 & 1,74 & 0,28 & 15 & 2,15 & 0,34 & 0,001 & T-Student \\
\hline $\mathrm{T} 11$ & 15 & 1,74 & 0,37 & 15 & 2,04 & 0,37 & 0,035 & T-Student \\
\hline $\mathrm{T} 12$ & 12 & 1,85 & 0,33 & 2 & 2,55 & 0,23 & 0,014 & T-Student \\
\hline $\mathrm{L} 1$ & 4 & 1,70 & 0,22 & 1 & 2,38 & - & 0,070 & T-Student \\
\hline $\mathrm{L} 2$ & 2 & 1,62 & 0,00 & 0 & - & - & - & - \\
\hline
\end{tabular}

do material de implante, e além de tudo em que nível isto seria mais acentuado. Quanto maior o ângulo c-a maior a posteriorização da aorta, chegando a obter distância aorta canal próximas a zero (0), demonstrando o contato entre a parede da artéria com a cortical da costela em muitos pacientes do nosso estudo.

No grupo de pacientes com EIA existe uma tendência a valores angulares maiores em níveis torácicos que coincidem com os níveis de ápice de deformidade. No grupo controle, entretanto, observamos valores médios do angulo c-a menores, a medida em que estudamos níveis mais caudais, além de uma menor variação entre eles. Assim, ocorrem valores estatisticamente diferentes $(p<0,05)$ em análise comparativa de valores médios de angulo c-a em níveis de T7 a T11, intervalo que compreende a maior parte das vértebras ou o nível de ápice de deformidade em portadores de EIA. Desta maneira, no grupo de pacientes portadores de EIA, quanto mais próximo do ápice da deformidade, maior o deslocamento lateral da aorta, que, entretanto em pacientes normais, encontra-se mais deslocada lateralmente em níveis mais craniais.

Bullmann et al. ${ }^{19}$ mostraram um migração da aorta de uma posição mais posterolateral antes da instrumentação na escoliose dextroconvexa torácica para uma posição mais anteromedial após a cirurgia.

Devido a rotação e distorção da vértebra a aorta invertida e a morfologia alterada do canal medular nos pacientes com escoliose, ${ }^{6,17,18}$ a inserção de parafusos na correção cirúrgica pode colocar o paciente em risco por uma lesão da aorta ou violação do canal medular ${ }^{20}$ o que foi demonstrado nos achados do ângulo c-a, tanto na inserção do implante por via torácica, como em nosso objetivo - via mediana posterior, em que um parafuso colocado em posição lateral apresentará maior chance de lesar a parede medial da aorta. Algumas complicações já foram descritas como invasão

\section{REFERÊNCIAS}

1. Weinstein SL, Dolan LA, Cheng JC, Danielsson A, Morcuende JA. Adolescent idiopathic scoliosis. Lancet. 2008:371(9623):1527-37.

2. Asher M, Lai SM, Burton D, Manna B, Cooper A. Safety and efficacy of Isola instrumentation and arthrodesis for adolescent idiopathic scoliosis: two- to 12-year follow-up. Spine (Phila Pa 1976). 2004;29(18):2013-23.

3. Arlet $\mathrm{V}$. Anterior thoracoscopic spine release in deformity surgery: a meta-analysis and review. Eur Spine J. 2000;9(Suppl 1):S17-23

4. Rosenthal D, Rosenthal R, de Simone A. Removal of a protruded thoracic disc using microsurgical endoscopy. A new technique. Spine (Phila Pa 1976). 1994;19(9):1087-91.

5. Khoo LT, Beisse R, Potulski M. Thoracoscopic-assisted treatment of thoracic and lumbar fractures: a series of 371 consecutive cases. Neurosurgery. 2002:51(Suppl 5):S104-17.

6. Maruyama T, Takeshita K, Nakamura K, Kitagawa T. Spatial relations between the vertebral body and the thoracic aorta in adolescent idiopathic scoliosis. Spine (Phila Pa 1976). 2004;29(18):2067-9.

7. Faciszewski T, Winter RB, Lonstein JE, Denis F, Johnson L. The surgical and medical perioperative complications of anterior spinal fusion surgery in the thoracic and lumbar spine in adults. A review of 1223 procedures. Spine (Phila Pa 1976). 1995;20(14):1592-9.

8. Szolar DH, Preidler KW, Steiner H, Riepl T, Flaschka G, Stiskal M, et al. Vascular complications in lumbar disk surgery: report of four cases. Neuroradiology. 1996;38(6):521-5.

9. Jendrisak MD. Spontaneous abdominal aortic rupture from erosion by a lumbar spine fixation device: a case report. Surgery. 1986:99(5):631-3.

10. Choi JB, Han JO, Jeong JW. False aneurysm of the thoracic aorta associated with an aorto-chest wall fistula after spinal instrumentation. J Trauma. 2001;50(1):140-3.

11. OhnishiT, Neo M, Matsushita M, Komeda M, Koyama T, Nakamura T. Delayed aortic rupture caused by an implanted anterior spinal device. Case report. J Neurosurg. 2001:95(Suppl 2):253-6.

12. Woolsey RM. Aortic laceration after anterior spinal fusion. Surg Neurol. 1986;25(3):267-8.

13. Heini P, Schöll E, Wyler D, Eggli S. Fatal cardiac tamponade associated with posterior spinal instrumentation. A case report. Spine (Phila Pa 1976). 1998;23(20):2226-30. do parafuso, ${ }^{21}$ laceração aórtica ${ }^{22}$ e pseudoaneurisma da aorta torácica ${ }^{10,23}$ em casos de instrumentação anterior.

Através da análise de TC das vértebras apicais (T8 ou T9) Sevastik et al. ${ }^{17}$ encontraram que a translação lateral média da aorta ao eixo médio do corpo vertebral aumentou no grupo com escoliose enquanto a distância vertical da aorta ao eixo médio do corpo vertebral diminuiu comparado com o grupo controle adulto. Liljenqvist et al. ${ }^{23}$ obteve o mesmo resultado medindo a distância entre a aorta para a vértebra sem comparar com a coluna normal. Sucato et al. $^{16}$ demonstraram que a aorta é localizada mais lateralmente e posteriormente em relação ao corpo vertebral nos pacientes com escoliose idiopática direita comparado àqueles normais. Adams ${ }^{24}$ descreveu que tanto nos pacientes com escoliose idiopática dextro-convexa quanto em pacientes normais, a aorta segue as vértebras pelo lado esquerdo do tórax. Em cadáveres com curvas para a esquerda são descritas posições da aorta com deslocamento para a direita. ${ }^{24}$ Os estudos que envolvem a posição da aorta em comparação com as vértebras na escoliose, apresentam uma dificuldade no que se refere aos pontos de referência anatômica, uma vez que a escoliose leva a uma alteração global da anatomia torácica. Nosso estudo demonstrou assim como a literatura, que com a rotação vertebral para a direita e com a lordose aumentada, ocorre um desvio posterior da aorta seguindo a linha mediana pelo lado esquerdo. Uma limitação apontada no nosso estudo é que o grupo controle não seguiu um padrão de sexo e idade semelhante ao estudado. Talvez a diferença de tamanho do tórax pudesse trazer-nos alguma alteração, porém não há motivo para que valores absolutos como tamanho, alterem a posição relativa da aorta, como já foi abordado por Maruyama et al. ${ }^{6}$ Podemos considerar isso quando analisamos os pacientes dentro de mesmos subgrupos com mesmo sexo e idade, e cujos resultados foram idênticos. O número de casos (n) também poderia ser considerada uma limitação do estudo, mas se encontra dentro da média de outros trabalhos.

\section{CONCLUSÃO}

Quanto mais próximo do ápice da deformidade, maior a posterolateralização da aorta através da analise comparativa do angulo c-a e distância canal-aorta. Deve-se durante o planejamento realizar uma analise criteriosa do posicionamento da aorta para evitar lesões precoces ou tardias resultantes do mau posicionamento dos parafusos.

Todos os autores declaram não haver nenhum potencial conflito de interesses referente a este artigo.
14. Lenke LG, Betz RR, Harms J, Bridwell KH, Clements DH, Lowe TG, et al. Adolescent idiopathic scoliosis: a new classification to determine extent of spinal arthrodesis. J Bone Joint Surg Am. 2001;83(8):1169-8116.

15. Cobb RJ. Outline for the study of scoliosis. Am Acad Orthop Surg 1948;5:261-75.

16. Sucato DJ, Duchene C. The position of the aorta relative to the spine: a comparison of patients with and without idiopathic scoliosis. J Bone Joint Surg Am. 2003:85-A(8):1461-9.

17. Sevastik $B$, Xiong $B$, Hedlund $R$, Sevastik J. The position of the aorta in relation to the vertebra in patients with idiopathic thoracic scoliosis. Surg Radiol Anat. 1996;18(1):51-6.

18. Qiu Y, He YX, Wang B, Zhu F, Wang WJ. The anatomical relationship between the aorta and the thoracic vertebral bodies and its importance in the placement of the screw in thoracoscopic correction of scoliosis. Eur Spine J. 2007:16(9):1367-72.

19. Bullmann V, Fallenberg E, Meier N, Fischbach R, Lerner T, Schulte T, Osada N, Liljenqvist $U$. The Position of the Aorta Relative to the Spine Before and After Anterior Instrumentation in Right Thoracic Scoliosis. Spine 2006;31(15):1639-760.

20. Matsuzaki H, Tokuhashi Y, Wakabayashi K, Kitamura S. Penetration of a screw into the thoracic aorta in anterior spinal instrumentation. A case report. Spine (Phila Pa 1976). 1993;18(15):2327-31

21. Sucato DJ, Kassab F, Dempsey M. Analysis of screw placement relative to the aorta and spinal canal following anterior instrumentation for thoracic idiopathic scoliosis. Spine (Phila Pa 1976). 2004:29(5):554-9.

22. Ahat $E$, Tuzun $H$, Bozkurt AK, Kaynak K, Erolcay HA. False aneurysm of the descending aorta due to penetrating injury. Injury. 1996;27(3):225-6.

23. Liljenqvist UR, Allkemper T, Hackenberg L, Link TM, Steinbeck J, Halm HF. Analysis of vertebral morphology in idiopathic scoliosis with use of magnetic resonance imaging and multiplanar reconstruction. J Bone Joint Surg Am. 2002;84(3):359-68.

24. Adams W. Lectures on the pathology and treatment of lateral and other forms of curvature of the spine. London: J Churchil \& Sons; 1865. 